\title{
Data Science and Machine Learning at Scale
}

\author{
Neel Sundaresan \\ Head of eBay Research Labs \\ nsundaresan@ebay.com
}

\begin{abstract}
Large Social Commerce Network sites like eBay have to constantly grapple with building scalable machine learning algorithms for search ranking, recommender systems, classification, and others. Large data availability is both a boon and curse. While it offers a lot more diverse observation, the same diversity with sparsity and lack of reliable labeled data at scale introduces new challenges. Also, availability of large data helps take advantage of correlational factors while requiring creativity in discarding irrelevant data. In this talk we will discuss all of this and more from the context of eBays large data problems.
\end{abstract}

